\title{
Opto-Chemical and Laser Properties of FLTX1, a Novel Fluorescent Tamoxifen Derivative, and its Potential Applications in Breast Cancer Photodynamic Chemotherapy
}

\author{
Mario Díaz ${ }^{1,6, *}$, Laura E. Scholz ${ }^{2}$, Jorge Marrero-Alonso ${ }^{1,6}$, Alicia Boto ${ }^{3,5}$, Raquel Marín ${ }^{4,6}$, Fernando \\ $\underline{\text { Lobo }^{3,6} \text {, Dácil Hernández }}{ }^{3,6}$, Angel Amesty ${ }^{7,8}$, Ana Estévez ${ }^{7,8}$, David Quinto-Alemany ${ }^{1,5}$, Ricardo \\ Puertas ${ }^{4}$, Fernando Lahoz ${ }^{2,5}$ \\ ${ }^{1}$ Dpto. Biología Animal, Universidad de La Laguna, 38200 La Laguna, Tenerife, Spain \\ ${ }^{2}$ Dpto. Física, IUdEA, Universidad de La Laguna, 38200 La Laguna, Tenerife, Spain \\ ${ }^{3}$ Instituto de Productos Naturales y Agrobiología, CSIC, 38206, La Laguna, Spain \\ ${ }^{4}$ Dpto. Físiología, Universidad de La Laguna, 38200 La Laguna, Tenerife, Spain \\ ${ }^{4}$ Dpto. Química Orgánica, Universidad de La Laguna, 38200 La Laguna, Tenerife, Spain \\ ${ }^{5}$ Unidad Asociada ULL-CSIC "Fisiología y Biofísica de la Membrana Celular en Enfermedades Neurodegenetarivas y \\ Tumorales" 38200 La Laguna, Tenerife, Spain \\ ${ }^{6}$ Instituto Universitario de Bioorgánica "Antonio González" Universidad de La Laguna, 38200 La Laguna, Tenerife, Spain
}

\begin{abstract}
Tamoxifen is the most common antiestrogen used in the chronic treatment of breast cancer. In these cells, it mainly binds to intracellular receptors (estrogen receptor alpha, ER $\alpha$ ) and antagonizes the binding of its cognate ligand, $17 \beta$-estradiol, thereby preventing uncontrolled hormonedependent cellular proliferation and growth. In the last decade, in our laboratories we have developed and characterized different tamoxifen derivatives, including a novel fluorescent tamoxifen conjugate: FLTX1. FLTX1 is formed by the covalent binding of tamoxifen to a common fluorescent biomarker NBD. This new prodrug was originally designed as a fluorescent biomarker to localize intracellular targets, which not only keeps the pharmacological activity of tamoxifen but also adds a luminescent functionality. Strikingly, the quantum efficiency of FLTX1 is so high that laser emission has been obtained as an emerging property. In this review, we will show its laser properties under three different configurations. First, as amplified spontaneous emission or mirrorless laser; second, through the evanescent field of WGMs of a ring resonator around an optical fiber; and finally as random laser in uterine tissues impregnated with the prodrug.

Further, we observed another emergent property for FLTX1: this molecule, but not tamoxifen alone or NBD, was able to generate reactive oxygen species (ROS) upon irradiation. This property is extremely interesting as FLTX1 might be used for photodynamic therapy. Under this paradigm, FLTX1 would act as a sensitizer in ER $\alpha$-overexpressing cells (which feature the most prevalent form of hormone-dependent breast cancer), causing cell death in ER $\alpha+$ cells but reducing damage to other non-cancer (healthy) cells or surrounding tissues. We show here time resolved fluorescence results that suggest molecular aggregations, which could be explanation of the subsequent generation of ROS. This is an original cancer therapy strategy that combines the pharmacological properties of a new tamoxifen derivative and its laser dye features with a highly selective photodynamic therapy.
\end{abstract}




\section{Introduction}

Tamoxifen is a selective modulator of estrogen receptors (SERMs) widely used as coadjuvant therapy in the treatment of breast cancer [1,2]. Tamoxifen is a triphenylethylene molecule that competes with the cognate ligand ( $17 \beta$-estradiol) for its binding to estrogen receptors (ER), and prevents the transcriptional activation of estrogen-dependent genes which control the proliferation of mammary gland cells [2]. Although it was first introduced in the late 1970s, it is still the first pharmacological strategy for hormone-sensitive breast cancers. In spite of its proven beneficial effects in the treatment of estrogen receptor positive breast cancers (the most prevalent form of hormone-dependent breast cancer), several side effects, some of them life-threatening, like womb cancer, endometrial cancer or thromboembolism, appear exacerbated in patients receiving chronic tamoxifen therapies $[3,4]$. The current view of these side-effects is that in most cases they are due to the interaction of tamoxifen with either canonical and/or non-canonical molecular targets, whose proper identification has been hampered because the lack of appropriate pharmacological tools. The existence of these adverse secondary effects has boosted many laboratories to search for either modifications in the tamoxifen molecule or novel SERMs structurally unrelated to tamoxifen, which could circumvent its undesirable effects during long-term therapies. In recent years, we have designed and developed a novel fluorescent tamoxifen derivative, FLTX1, which not only allows researchers tracking canonical (i.e. ER) and non-canonical intracellular targets, but also retains most pharmacological properties of tamoxifen (specific ER binding, antagonism of ER-mediated transcriptional activation and cell proliferation), while lacks the trophic worrisome effects of tamoxifen in the uterus [5]. Beyond its pharmacological properties, FLTX1 is endowed with emergent opto-chemical properties of which tamoxifen molecule is devoid [6-8]. Thus, positive gain media based on FLTX1 were prepared and laser emission was demonstrated under optical pulsed excitation. In this article we will review the main properties of FLTX1 with special focus on its laser properties and its potential application as specific site-directed photosensitizer.

\section{FLTX1}

FLTX1 was developed as a fluorescent derivative of the anticancer drug tamoxifen. It was initially formed from tamoxifen (1, Scheme in Figure $1 \mathrm{~A}$ ) by demethylation (to give 2 ) and coupling to the fluorophore nitro-2-1,3-benzoxadiazol-4-yl group (NBD), by reaction with commercial NBD-Cl. Alternatively, since tamoxifen is an expensive substrate, a second route was developed from lowcost substrates. Thus, McMurray coupling between ketones (4) and (5) gave the tamoxifen core (6), which was transformed in two steps into demethyltamoxifen $(2, R=M e)$. This second route allows the generation of other tamoxifen derivatives, by using different $\mathrm{R}$ groups.

The resulting product, FLTX1, dissolved in methanol, exhibits the fluorescent spectral features illustrated in Figure 1A (right panel). FLTX1 is an optically active molecule, which shows a broad absorption band centered at about $475 \mathrm{~nm}$. It has full width at half maximum (FWHM) around 65 $\mathrm{nm}$. When irradiated at $475 \mathrm{~nm}$, a broad fluorescence emission band (FWHM $\approx 80 \mathrm{~nm}$ ) with maximum emission at about $550 \mathrm{~nm}$.

\section{Pharmacological properties of FLTX1 at a glance}

An in-depth study on the pharmacological properties of FLTX1 was published by our group in 2013 [5]. A number of different in vitro and in vivo methodological approaches were used to demonstrate the usefulness of FLTX1 as a prodrug, from confocal laser scanning microscopy and transcriptional modulation of gene expression in cultured human breast cancer cells (MCF-7 cells) to long-term administration in mice. 
A)

\section{B)}

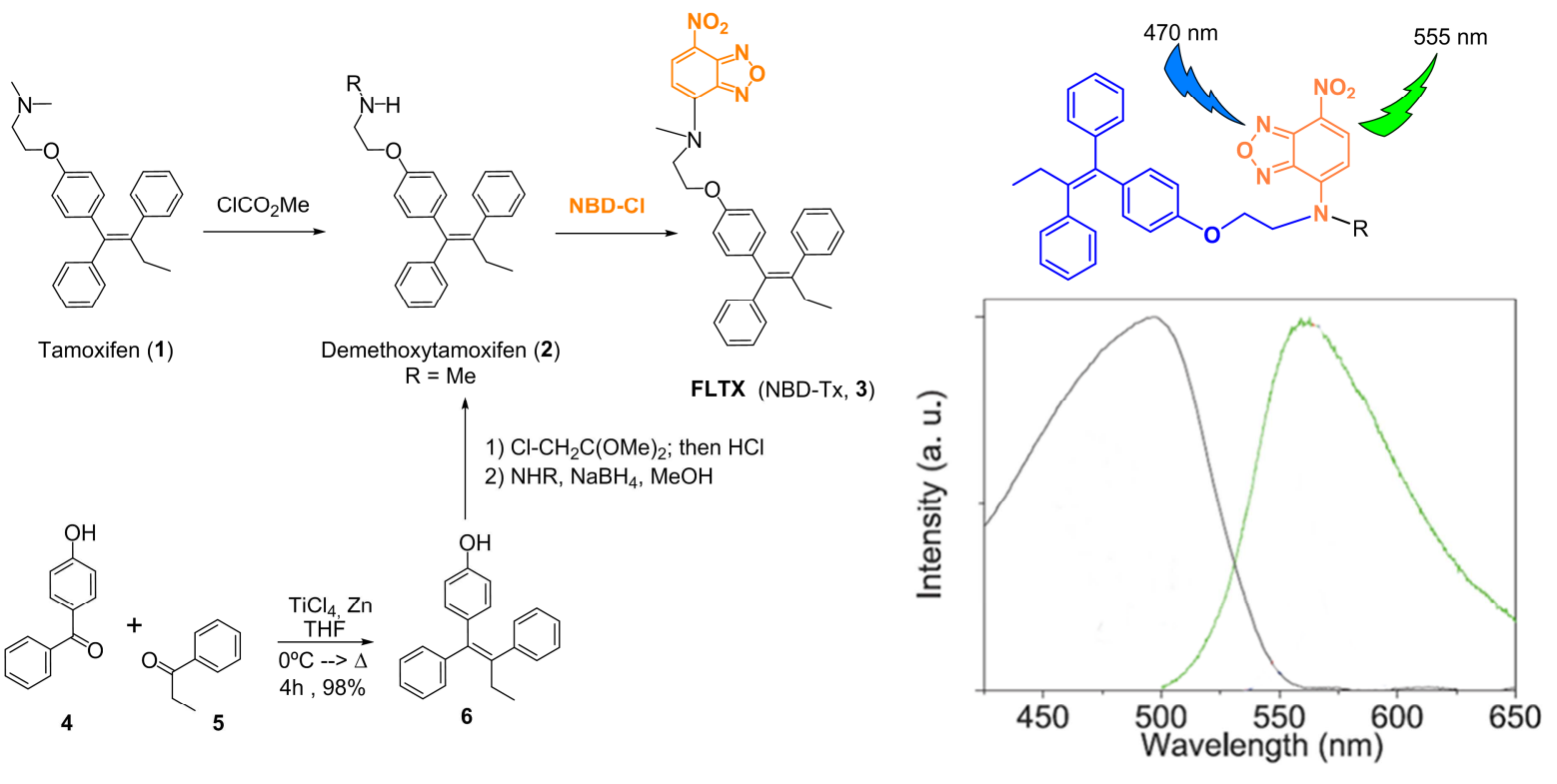

FLTX1

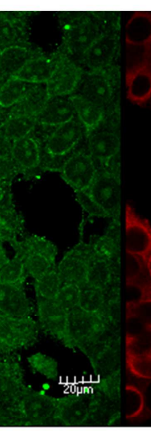

.

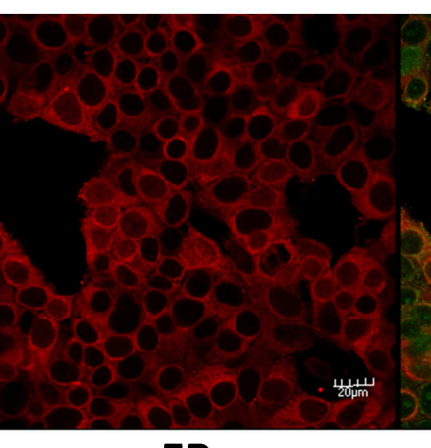

$E R \alpha$

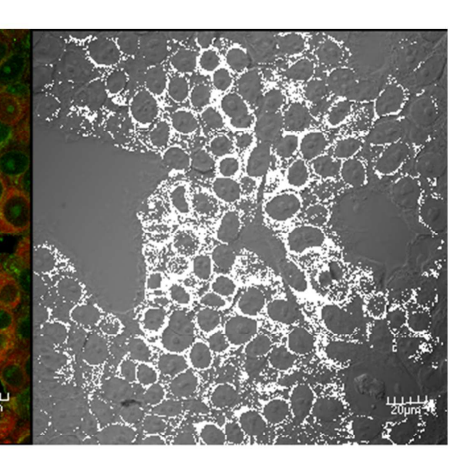

Transmission

Figure 1. A) Synthesis (left panel) and spectral characteristics (right panel) of FLTX1. B). FLTX1 and ER $\alpha$ colocalize in MCF7 breast cancer cells. To investigate the potential colocalization of these two molecules, we performed immunocytochemical assays using standard protocols described previously [5]. MCF7 cells were incubated with FLTX1 (green fluorescence), followed by incubation with MC20, an anti-ER $\alpha$ specific antibody (red fluorescence). The results obtained by confocal microscopy revealed that both fluorescent signals (merged images FLTX1 and ER $\alpha$ in panel 'Merge'). In the rightmost panel, transmission image overlapped with white spots corresponding to FLTX1 and MC20 colocalization.

\section{FTLX1 in vitro}

In MCF-7 cells, FLTX1 binds intracellular targets which colocalizes with estrogen receptors [9]. A representative example of FLTX1 and ER labelling is shown in Figure 1B. FLTX1 binding was dosedependent and could be totally displaced by tamoxifen (and partially by $17 \beta$-estradiol), also in a concentration-dependent manner [5]. Quantitation of colocalization between FLTX1 and MC20 antibody (which specifically recognises $\mathrm{ER} \alpha$ ) was around $71 \%$, mostly at the perinuclear space (Fig. 1b). The inability of $17 \beta$-estradiol to totally displace FLTX1 labelling, together with the fact that nearly $30 \%$ of FLTX1 labelling do not colocalize with ER $\alpha$ in MCF7 cells, reinforces the notion that tamoxifen interacts with targets other than $E R \alpha$, some of which likely underlie some undesirable side effects of tamoxifen [10-13]. Precise determination of binding affinity of FLTX1 for ER $\alpha$ was determined in competition experiments in rat uterine cytosolic extracts (a classical preparation highly enriched in $E R \alpha$ ) showed that the $\mathrm{IC}_{50}$ values (the drug concentration that causes $50 \%$ of maximal competition) for FLTX1 and tamoxifen were $87.5 \mathrm{nM}$ and $123.4 \mathrm{nM}$, respectively. Thus, 
assuming a Relative Binding Affinity (RBA) value of 100 for tamoxifen, the estimated value for FLTX1 was 141.01, indicating a slightly increased affinity of FLTX1 for ER than tamoxifen.

\section{Docking of FTLX1 on ER $\alpha$}

In order to get a deeper insight on the reasons for the higher affinity of FLTX1 over tamoxifen, we performed in silico docking studies. The results are shown in Figure 2. The X-ray protein coordinates were extracted from the Protein Data Bank (PDB code 3ERT). The PDB structure was prepared for docking using the Protein Preparation Workflow (Schrodinger, LLC, New York, NY, 2017) accessible from within the Maestro program. The substrate and water molecules were removed beyond $5 \AA$, bond corrections were applied to the cocrystallized ligands and an exhaustive sampling of the orientations of groups was performed. Finally, the receptor was optimized in Maestro 11.4 by using OPLS2005 force field. The receptor grids were generated using the prepared protein, with the docking grids centered for the ligand bound to the receptor. The ligands were docked using the extra precision mode (XP) [14] without using any constraints. The generated ligand poses were evaluated with empirical scoring function, GlideScore a modified version of ChemScore [15]. GlideScore implemented in Glide, was used to estimate binding affinity and rank ligands [16]. The XP Pose Rank was used to select the best-docked pose for each ligand.

A)

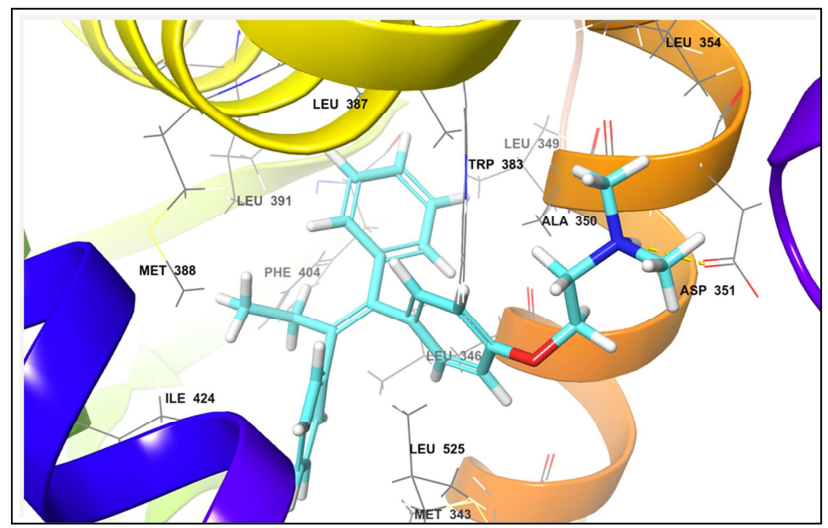

B)

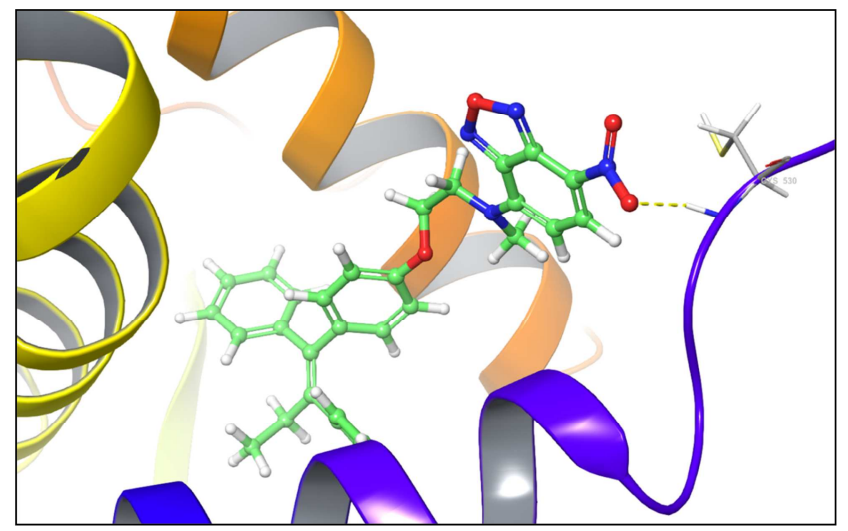

Figure 2. Docking of A) Tamoxifen and B) FLTX1 into the binding site of ER $\alpha$ (PDB 3ERT).

As we can see in Figure 2, the introduction of the NBD moiety to the tamoxifen (Figure 2A) produces a new hydrogen bond interaction between the $-\mathrm{NO}_{2}$ group and Cys 530 of helix 12 (XPGlide score $11.55 \mathrm{Kcal}^{\mathrm{mol}}{ }^{-1}$ ) (Figure 2B). This interaction seems to be more effective than those that tamoxifen establishes between the protonated nitrogen and Asp351 (XPGlide score $-10.83 \mathrm{Kcal}^{\mathrm{mol}}{ }^{-1}$ ). This new interaction with the helix 12 in ER $\alpha$ explains the higher affinity of FLTX1 over tamoxifen.

\section{FTLX1 in vivo}

In vivo, FLTX1 was able to inhibit MCF-7 cell growth. This cell line is characterized by the fact that its growth and proliferation is dependent on the presence of estrogens. Our findings showed that FLTX1 was able to reduce estrogen-induced cell proliferation at concentrations as low as $3.0 \mu \mathrm{M}$ (which is even lower than the concentration found in patients receiving tamoxifen coadjuvant therapies). As estrogen-dependent cell proliferation is dependent on the activation of estrogen- 
responsive genes, we also performed genetic analyses on the effects of FLTX1 and tamoxifen on transcriptional activation of these genes. We used a strategy based on the manipulation of a specific promotor region (where activated ER binds to the DNA) named estrogen responsive element (ERE). We used a genetic construct where the luciferase gene was engineered to be transcribed under the control of ERE. Thus, whenever estrogens activate DNA transcription, the luciferase protein would be expressed and will emit an amount of luminescence, whose intensity is graded according to the degree of transcriptional activation. The construct was transfected into MCF-7 and TD47 cell lines.

The difference between tamoxifen and FLTX1 was dramatic. Thus, while tamoxifen alone was able to induce transcriptional activation of luciferase, particularly at low concentrations (between 0.01 and $1 \mu \mathrm{M})$, FLTX1 was completely devoid of this property in the whole range of concentrations assayed. However, as expected, both compounds exhibited similar antiestrogenic behaviours in inhibiting estradiol-induced transcriptional activation [5].

The last set of in vivo experiments was aimed to address the actions of FLTX1 in the uterus. These experiments were particularly interesting, since the uterus is the locus of some of the most important side effects of tamoxifen. Indeed, the major concern in the use of tamoxifen as adjuvant therapy in the treatment of breast cancer is the increased risk of developing uterine cancer and endometriosis $[3,4]$. In mice, tamoxifen causes a potent uterotrophic effect, being as estrogenic as $17 \beta$-estradiol itself [17]. Tamoxifen caused hypertrophy, endometrial hyperplasia, augmented uterine weight and increased stromal gland number. Strikingly, under similar experimental paradigms, the effects of FLTX1 were completely negligible, behaving as the placebo [5].

\section{FLTX1 and its optical properties}

\section{Fluorescence decay properties}

The decay of the fluorescence was measured under pulsed excitation at $475 \mathrm{~nm}$ and detection at $550 \mathrm{~nm}$ as a function of the concentration of the FLTX1 solution using acetone as solvent. The decay curves were fitted to a double exponential decay of the form:

$$
I(t)=A_{1} e^{\left(-\frac{t}{\tau_{1}}\right)}+A_{2} e^{\left(-\frac{t}{\tau_{2}}\right)}
$$

Where $\tau_{1}$ and $\tau_{2}$ are the decay constants and $A_{1}$ and $A_{2}$ the pre-exponential factors. The best fitting was obtained for short and long decay constants of $\tau_{1}=0.3 \mathrm{~ns}$ and $\tau_{2}=7 \mathrm{~ns}$, respectively. The preexponential factor of the short decay component was several orders of magnitude higher than the long decay one and, in all the cases, the ratio of $A_{1}$ was more than $99 \%$. Moreover, the contribution of the long decay component decreases as the concentration increases and it is negligible at the highest concentration of $10 \mathrm{mM}$, (Figure 3). This behavior can be related to the tendency of FLTX1 to aggregate in the form dimers. The lifetime of dimers is expected to be lower than that of isolated molecules due to the interaction of the molecules in the aggregated form and the formation of triplet excited states, which introduce additional relaxation paths. Therefore, the short decay component would be assigned to dimers, while the short one to isolated molecules, which are more abundant at lower concentrations. The aggregation into dimers might have a strong influence in the production of ROS, as it will be shown later. 


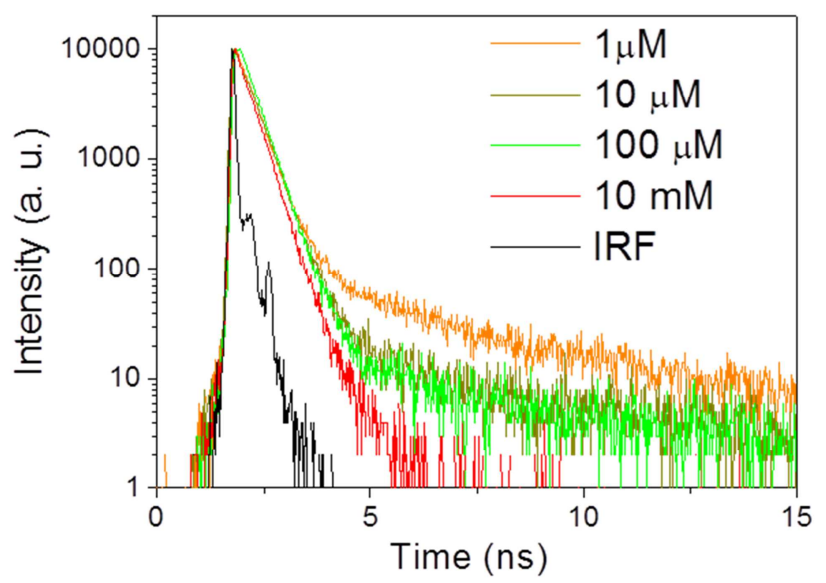

Figure 3. Decay curves at different concentration of FLTX1 dissolved in acetone. IRF: Instrumental Response Function

We were interested in the determination of the capability to produce laser emission out of the fluorescent drug complex, FLTX1. Consequently, we designed three different laser configuration setups.

\section{Amplified Spontaneous Emission}

An easy and convenient way to check the laser properties of organic materials is trough Amplified Spontaneous Emission (ASE) [18-21]. ASE is a cooperative luminescent effect, which occurs by successive stimulated emission of radiation from the molecules in the excited state in the presence of photons with an energy that coincides with the energy gap between the excited and the fundamental state of the molecules. ASE is observed when optical gain in the active medium is higher than the optical losses through it. It is sometimes referred to as Mirrorless Laser because it occurs without any mirror nor resonator to provide optical feedback [22]. In order to prove if ASE can be produced in a solution of FLTX1, an OPO pulsed laser tuned at $475 \mathrm{~nm}$ was used as the excitation source [8]. The pump beam was focused on a stripe line at the front surface of the probe cuvette using a cylindrical lens (Figure 4A). At relatively low pump power the typical broad emission band of FLTX1 was detected. However, when the pump power was increased above a certain pump threshold the emission spectrum narrowed and the emission intensity increased abruptly (Figure 4B). These are the two characteristic features of ASE which confirm that the medium had a positive optical gain [20-22]. The pump power threshold and the ASE efficiency were measured as a function of the FLTX1 concentration (Figure 4C). The highest efficiency was found for the $10 \mathrm{mM}$ FLTX1 solution with a relatively low pump threshold of $7.5 \mu \mathrm{J} / \mathrm{mm}^{2}$. 

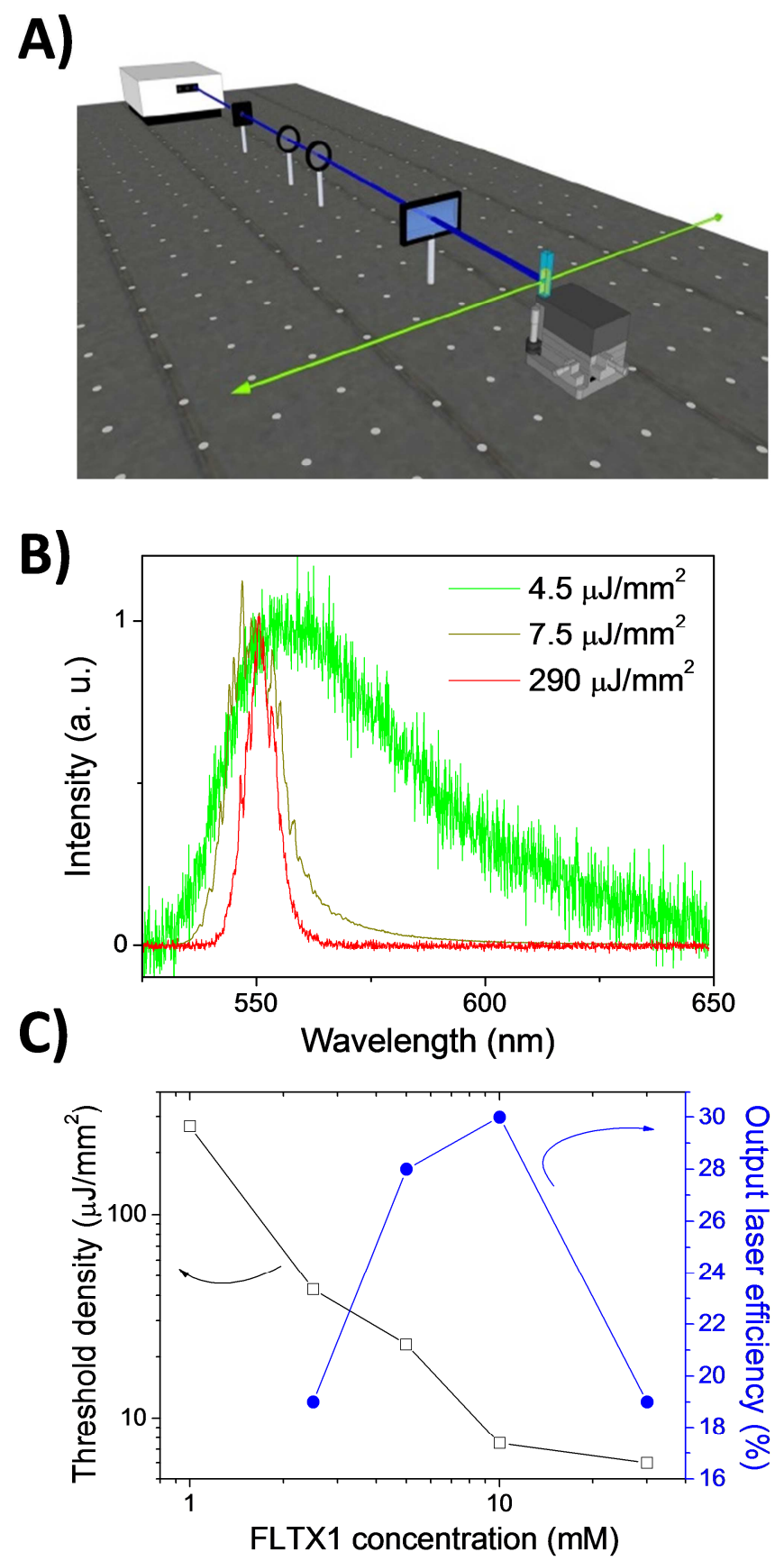

Figure 4. Amplified Spontaneous Emission of FLTX1. A) Experimental setup; B) Narrowing of the emission spectrum as a function of the pump density; C) ASE threshold and efficiency as a function of the FLTX1 concentration.

Once we had shown that the radiative emission efficiency of FLTX1 was enough to produce optical gain, we planned to obtain laser emission using a microresonator to provide the required optical feedback.

\section{Whispering Gallery Mode Laser}

Spherical or cylindrical dielectric microresonators have shown the ability to support whispering gallery modes (WGM) lasers without external mirrors. They have found interesting applications in 
optofluidic devices $[23,24]$. A simple way to obtain WGM laser emission was proposed in reference [25]. These authors used an optical fiber as a cylindrical microresonator and it was surrounded by the optical gain medium. The evanescent wave of the WGM of the microresonator couples the gain molecules that surround the resonator and stimulate the emission of radiation. In our case, a segment of silica $125 \mu \mathrm{m}$ diameter optical fiber was dipped into the FLTX1 solution, resting vertically against the cuvette front wall. A cylindrical lens focused the pump beam on the cuvette front face to form a horizontal line (300 ? $\mathrm{m}$ long by 210 ? $\mathrm{m}$ wide), which homogeneously illuminated the optical fiber and surrounding dye solution. Clock-wise and anticlock-wise WGMs couple to the cuvette wall through the contact area and produce laser beams in both directions. As the pump power is increased the broad emission spectrum suddenly becomes a series of narrow lines at a given threshold (Figure 5). In addition to this, the emission intensity abruptly increases. Both the sudden increase of the emission intensity and the narrow lines of the emission spectrum are evidences of laser oscillation [7]. The observed pump energy density threshold for WGM laser emission was 19 $\mu \mathrm{J} / \mathrm{mm}^{2}$.
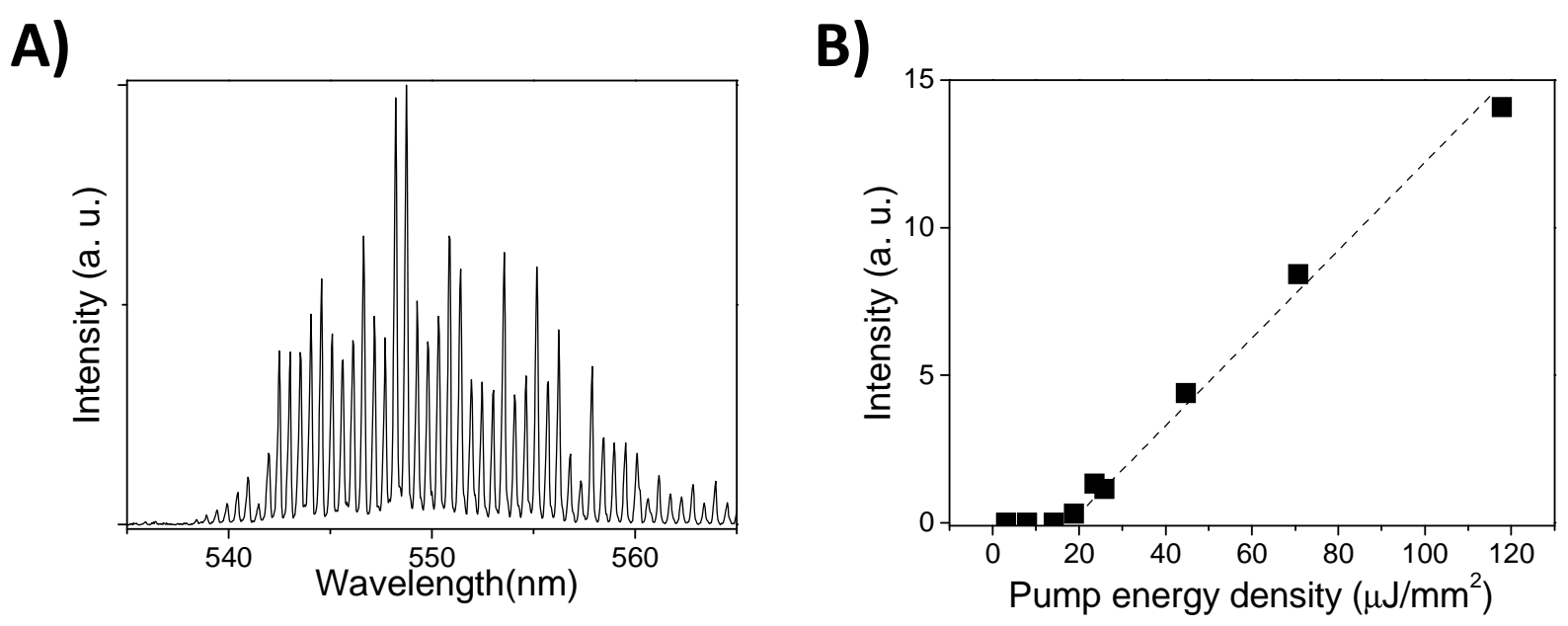

Figure 5. Whispering Gallery Mode Laser of FLTX1. A) Laser emission spectrum of the WGMs; B) Emission intensity as a function of pump energy density.

\section{Random Laser}

In the last two sub-sections, we have shown the ability of FLTX1 to provide laser emission without (ASE) or with (WGM) optical feedback using a solution of the fluorescent drug in a cuvette. However, we wanted to demonstrate the laser emission capability of the prodrug in biological systems. For that purpose, we tested random laser ( $R L$ ) emission because it happens when the optical gain medium produces scattering of the light. RL occurs without external resonators, since optical feedback, necessary for laser oscillation, is provided by a random collection of scatters $[26,27]$. RL has been reported in biological tissues impregnated with a concentrated laser dye solution [28-31]. Therefore, we planned to use FLTX1 as a fluorescent biomarker to embed tissues, such as uterine tissues, in which $E R \alpha$, the main molecular targets of FLTX1, are particularly abundant [9].

Uterine tissues from mice and rats were fixed with paraformaldehyde and processed as previously described [9]. After staining, preparations were mounted in glass slides with a drop of $10 \%$ glycerol and covered with coverslips for RL studies.

The OPO pulsed pump laser was tuned at the maximum of the FLTX1 absorption band. A cylindrical lens focused the pump beam on the tissue with normal incidence to form a homogeneous 
horizontal line $4 \mathrm{~mm}$ long and $210 \mu \mathrm{m}$ wide.

At low pump power, the characteristic broad luminescence band of FLTX1 centered at about $550 \mathrm{~nm}$ was detected. However, under high pump power excitation, the emission spectrum dramatically narrowed and the emission intensity sharply increased. These are the two typical features of a transition from spontaneous emission to laser emission. Nevertheless, two different types of spectra were recorded depending on the excitation region of the sample. In the first case, the FWHM dropped to about 12-15 nm at the pump threshold (Figure 6A). However, in the second case, the FWHM dropped to about $0.5 \mathrm{~nm}$, which was limited by the spectral resolution of the acquisition setup (Figure 6B). The first type of laser emission corresponded to incoherent RL, while the second to coherent RL. The appearance of incoherent or coherent RL depended on the region of the tissue under optical excitation. A change in the position of the irradiated tissue by a few hundreds of micrometers could be enough to vary from coherent $R L$ to incoherent $R L$. In the case of coherent $R L$ the stimulated emitted light wave experiences phase coherence, which produces additive wave interference, giving rise to the sharp resonances observed in the coherent RL spectra [32]. The inhomogeneities of the tissue act as scatters of the light (Figure $6 \mathrm{C}$ ). Those regions with a high density of scatters could give rise to close loops of the scattered light, which experiences optical amplification due to stimulated emission, and would produce the narrow peaks of coherent RL. The fact that some regions show incoherent $\mathrm{RL}$, while others coherent one could be related to the different density of scattering centers in the tissue.

In both incoherent and coherent RL, the pump density threshold was about $120-140 \mu \mathrm{J} / \mathrm{mm}^{2}$, depending on the region of the tissue. A detailed analysis of the spectral position of the RL coherent peaks could provide information about the length of the closed loops of the stimulated emission light paths [28]. In our case, we deduced a closed loop length of about $18 \mu \mathrm{m}$, which is in close according to average cell diameter. Taking into account that typical sizes of the cells in uterine tissues under study were about 10-20 $\mu \mathrm{m}$ long, RL could occur inside a single cell. However, this possibility has not been proved yet. 

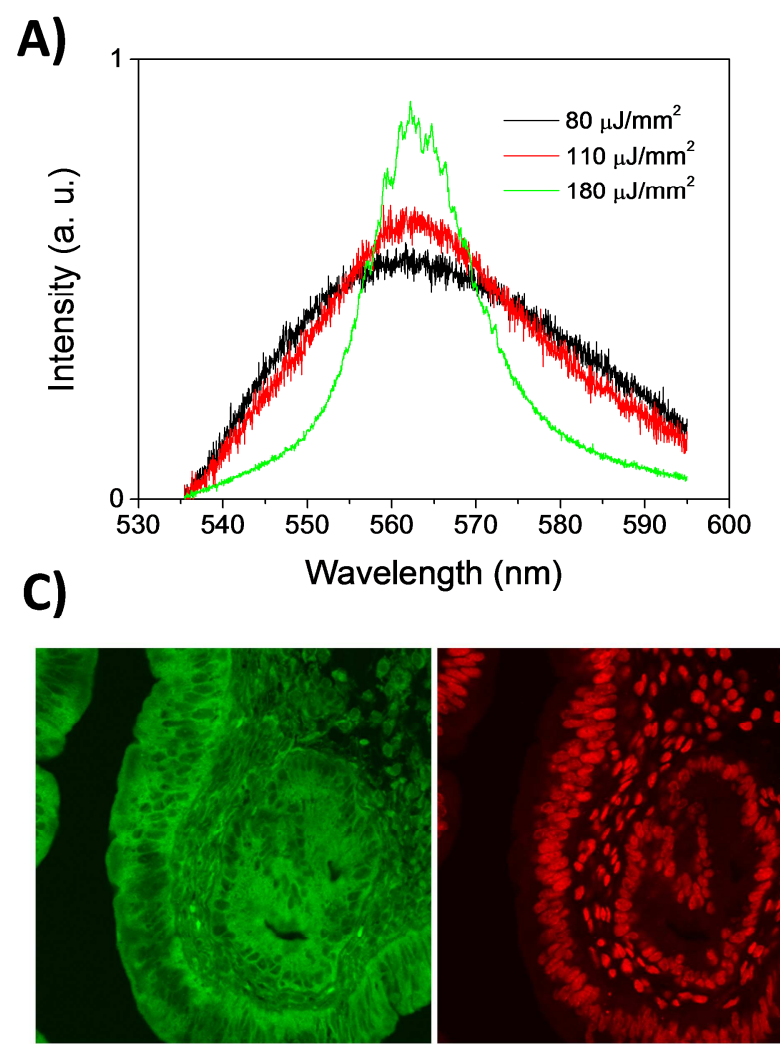

FLTX1

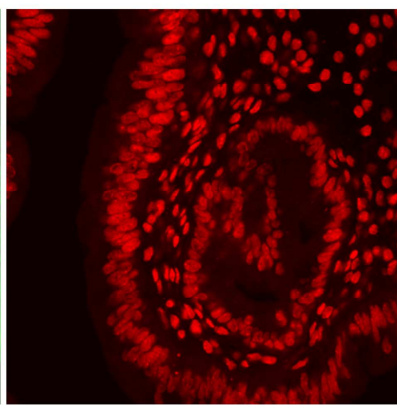

Propidium iodide
B)

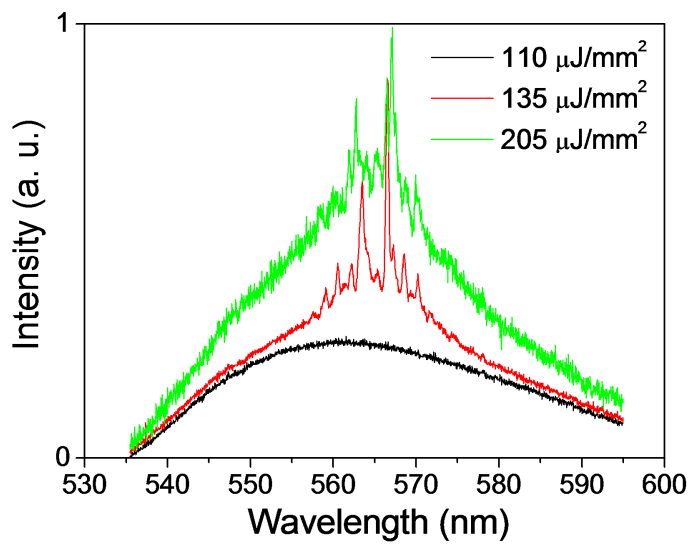

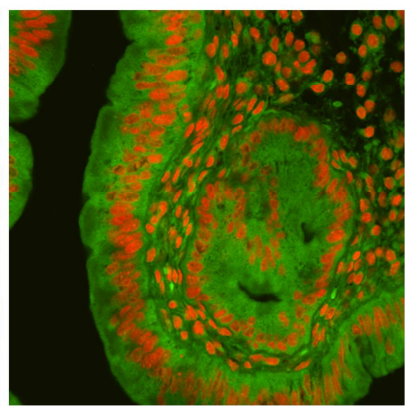

Merge

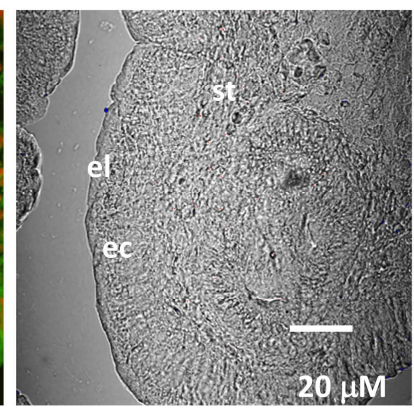

Transmission

Figure 6. Random Laser properties of FLTX1. Emission spectra as a function of pump density showing the transition from spontaneous emission to $A$ ) incoherent $R L$ and $B$ ) coherent $R L$. C) Confocal microphotographs of mouse uterine wall showing the different cell layers (el: epithelial lining; st: stroma; ec: columnar epithelial cells; mg: mucous gland) labelled with FLTX1 solutions. Illustrated are, from left to right, FLTX1, propidium iodide, merged and phase contrast transmission images.

\section{FLTX1 as photosensitizer}

In order to evaluate FLTX1 as a photosensitizer drug, and its ability to produce reactive oxygen species or radicals, a colorimetric tetrazolium/formazan assay was implemented, using nitro blue tetrazolium (NBT). NBT is a water-soluble, yellow compound that upon reaction with oxidizing radicals is transformed into a chromogenic formazan dyes with a characteristic, intense blue colour [33]. Thus, when solutions of FLTX1 and NBT are irradiated, reactive species produced by FLTX1 oxidize NBT and yield the blue formazan dye, which displays a broad absorption band at 500-600 $\mathrm{nm}$. Under this paradigm, the amount of oxidized formazan will depend on the photosensitizer activity of FLTX1.

In our assays we measured changes of formazan absorbance produced by $1 \mathrm{~mL}$ samples of $50 \mu \mathrm{M}$ FLTX1 and NBT in DMSO (dimethyl sulfoxide) irradiated at $473 \mathrm{~nm}$ during 0, 4, 8, 12, 16 and 20 minutes. The curves resulting from subtraction of the absorbance at $t=0$ (control) are shown in Figure 7A (left panel). The absorbance spectra due to formazan generation between 500 and 600 $\mathrm{nm}$ was observed at all times, indicating ROS generation in an irradiation time-dependent manner. The increase in absorbance is totally attributable to the presence of FLTX1, since in the presence of NBT alone, only small changes of absorbance in the range 500-600 nm were detected in the absence of FLTX1 (Figure 7, right panel) 

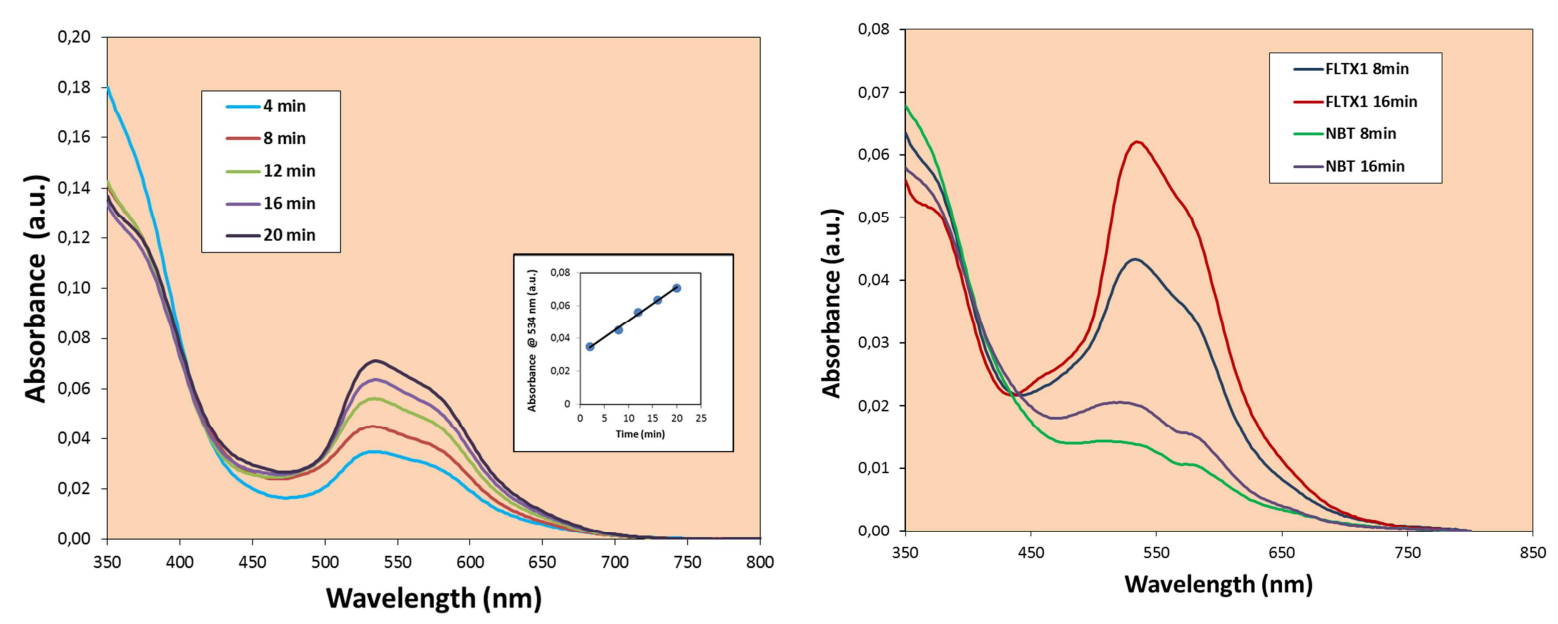

Figure 7. A) Absorbance spectra of formazan produced after irradiating a $50 \mu \mathrm{M}$ FLTX-NBT solution @ 475 $\mathrm{nm}$ at different times (Left). B) Typical absorbance curves with respect to irradiated NBT as control (Right).

\section{Conclusions}

In the present article, we have delineated some biological, pharmacological and optochemical characteristics of a novel anticancer progrud developed in our laboratories. This compound, named FLTX1, is endowed with excellent properties so as to be considered a potential chemotherapeutic agent against estrogen receptor positive breast cancer. FLTX1 specifically binds estrogen receptors and antagonizes estrogen-dependent cell growth, while lacks main adverse effects on uterus exhibited by the parent molecule: tamoxifen. Additionally, FLTX1 exhibits surprising optical properties and behaves a laser dye, which can be demonstrated under different configurations, namely ASE, WGM and RL. Strikingly, FLTX1 also displays an emergent property as photosensitizer in situ, generating reactive species when irradiated at wavelengths which induce laser emission.

Acknowledgements. Supported by Research grant SAF2014-61644-EXP from MINECO (Spain) to M.D. Partially supported by Agencia Estatal de Investigación (AEI, grant ref. MAT2016-79866-R to F.L.), SAF2015-65113-C2-1-R and SAF2017-84454-R (from MINECO) to A.E. and R.M., respectively. $\mathrm{DH}$ and DQA were hired by Universidad de La Laguna. FL was recipient of contract from Torres Quevedo Programme (MINECO) and incorporated in BIOSIGMA SL (Spain)

\section{References}

[1] C.K. Osborne, H. Zhao, S.A. Fuqua, Selective estrogen receptor modulators: structure, function, and clinical use, J. Clin. Oncol. 18 (2000) 3172-3186.

[2] J.I. MacGregor, V.C. Jordan, Basic guide to the mechanisms of antiestrogen action, Pharmacol. Rev. 50 (1998) 151-196.

[3] J. Iqbal, O.M. Ginsburg, T.D. Wijeratne, A. Howell, G. Evans, I. Sestak, S.A. Narod, Endometrial cancer and venous thromboembolism in women under age 50 who take tamoxifen for prevention of breast cancer: a systematic review, Cancer Treat. Rev. 38 (2012) 318-328.

[4] C. Dong, L. Chen, Second malignancies after breast cancer: the impact of adjuvant therapy, Mol. Clin. Oncol. 2 (2014) 331-336. 
[5] J. Marrero-Alonso, A. Morales, B. García-Marrero, A. Boto, R. Marín, D. Cury, T. Gómez, L. Fernández-Pérez, F. Lahoz, M. Díaz, Unique SERM-like properties of the novel fluorescent tamoxifen derivative FLTX1, Eur. J. Pharm. Biopharm. 85 (2013) 898-910.

[6] A. Morales, R. Marín, J. Marrero-Alonso, A. Boto, M. Díaz, Colocalization of Estrogen Receptors with the Fluorescent Tamoxifen Derivative, FLTX1, Analyzed by Confocal Microscopy, Methods Mol. Biol. 1366 (2016) 163-173.

[7] F. Lahoz, C. J. Oton, D. López, J. Marrero-Alonso, A. Boto, M. Díaz, Whispering gallery mode laser based on antitumor drug-dye complex gain medium, Opt. Lett. 37 (2012) 4756-4758.

[8] F. Lahoz, C. J. Oton, D. López, J. Marrero-Alonso, A. Boto, M. Díaz, High efficiency amplified spontaneous emission from a fluorescent anticancer drug-dye complex, Org. Electron. 14 (2013) 1225-1230.

[9] F. Lahoz, I.R. Martín, M. Urgellés, J. Marrero-Alonso, R. Marín, C. J. Saavedra, A. Boto, M. Díaz, Random laser in biological tissues impregnated with a fluorescent anticancer drug, Laser Phys. Lett. 12 (2015) 045805.

[10] M. Díaz, Triphenylethylene antiestrogen-induced acute relaxation of mouse duodenal muscle. Possible involvement of $\mathrm{Ca}^{2+}$ channels, Eur. J. Pharmacol. 445 (2002) 257-266.

[11] J. Marrero-Alonso, B. García Marrero, T. Gómez, M. Díaz, Functional inhibition of intestinal and uterine muscles by non-permeant triphenylethylene derivatives, Eur. J. Pharm. 532 (2006) 115-127.

[12] P. de Médina, G. Favre, M. Poirot, Multiple targeting by the antitumor drug tamoxifen: a structure-activity study, Curr. Med. Chem. Anticancer Agents 4 (2004) 491-508.

[13] M. Díaz, B. García-Marrero, J. Marrero-Alonso, R. Marín, T. Gómez, R. Alonso, Cellular and molecular basis for acute nongenomically-mediated actions of SERMs, in: A. Cano, J. Calaf i Alsina, J.L. Duenas-Diez (Eds.), Selective Estrogen Receptor Modulators: A New Brand of Multitarget Drugs, Springer-Verlag, Berlin, Heidelberg, 2006, pp. 79-102.

[14] R.A. Friesner, R.B. Murphy, M.P. Repasky, L.L. Frye, J.R. Greenwood, T.A. Halgren, P.C. Sanschagrin, D.T. Mainz, Extra precision glide: docking and scoring incorporating a model of hydrophobic enclosure for protein-ligand complexes, J. Med. Chem. 49 (2006) 6177-6196.

[15] M.D. Eldridge, C.W. Murray, T.R. Auton, G.V. Paolini, R.P Mee, Empirical scoring functions: I. The development of a fast empirical scoring function to estimate the binding affinity of ligands in receptor complexes, J. Comput. Aided Mol. Des. 11 (1997) 425-445.

[16] R.A. Friesner, J.L. Banks, R.B. Murphy, T.A. Halgren, J.J. Klicic, D.T. Mainz, M.P. Repasky, E.H. Knoll, M. Shelley, J.K. Perry, D.E. Shaw, P. Francis, P.S. Shenkin, Glide: a new approach for rapid, accurate docking and scoring. 1. Method and assessment of docking accuracy, J. Med. Chem. 47 (2004) 1739-1749.

[17] S.D. Lyman, V.C. Jordan, Metabolism of tamoxifen and its uterotrophic activity, Biochem. Pharmacol. 34 (1985) 2787-2794.

[18] A.E. Siegman, Lasers, University Science Books, Mill Valley, California, 1986.

[19] I.D.W. Samuel, G.A. Turnbull, Organic semiconductor lasers, Chem. Rev. 107 (2007) 1272-1295.

[20] E.M. Calzado, P.G. Boj, M.A. Díaz-García, Amplified spontaneous emission properties of semiconducting organic materials, Int. J. Mol. Sci. 11 (2010) 2546-2565.

[21] A. Costela, O. García, L. Cerdán, I. García-Moreno, R. Sastre, Amplified spontaneous emission and optical gain measurements from pyrromethene 567-doped polymer waveguides and quasiwaveguides, Opt. Express 16 (2008) 7023-7036.

[22] P. Yang, G. Wirnsberger, H.C. Huang, S.R. Cordero, M.D. McGehee, B. Scott, T. Deng, G.M. Whitesides, B.F. Chmelka, S.K. Buratto, G.D. Stucky, Mirrorless lasing from mesostructured waveguides patterned by soft lithography, Science 287 (2000) 465-467. 
[23] H. Schmidt and A. R. Hawkins, The photonic integration of non-solid media using optofluidics, Nat. Phot. 5 (2011) 598-604.

[24] Z. Li and D. Psaltis, Optofluidic dye lasers, Microfluid Nanofluid 4 (2008) 145-158.

[25] H. J. Moon, Y. T. Chough, and A. Kyungwon, Cylindrical microcavity laser based on the evanescent-wave-coupled gain, Phys. Rev. Lett. 85 (2000) 3161-3164.

[26] V. S. Letokhov, Generation of light by a scattering medium with negative resonance absorption, Sov. Phys. - JETP 26 (1968) 835-840.

[27] N. M. Lawandy, R. M. Balachandran, A. S. L. Gomes, E. Sauvain, Laser action in strongly scattering media, Nature 368 (1994) 436-438.

[28] R. C. Polson, Z. V. Vardeny, Random lasing in human tissues, Appl. Phys. Lett. 85 (2004) 12891291.

[29] Q. Song , S. Xiao, Z. Xu, J. Liu, X Sun,V. Drachev, V. M. Shalaev, O. Akkus, Y. L. Kim, Random lasing in bone tissue, Opt. Lett. 35 (2010) 1425-1427.

[30] J. C. Briones-Herrera, N. Cuando-Espitia, F. M. Sánchez-Arévalo, J. Hernández-Cordero, Evaluation of mechanical behavior of soft tissue by means of random laser emission, Rev. Sci. Instrum. 84 (2013) 104301.

[31] D. Zhang, G. Kostovski, C. Karnutsch, A. Mitchell, Random lasing from dye doped polymer within biological source scatters: the pomponia imperatorial cicada wing random nanostructures, Org. Electron. 13 (2012) 2342-2345.

[32] H. Cao, J. Y. Xu, Y. Ling, A. L. Burin, E. W. Seeling, X. Liu, and R. P. H. Chang, "Random lasers with coherent feedback," IEEE J. Sel. Top. Quant. Electr. 9 (2003) 111-119.

[33] B. Halliwell, J.M.C. Gutteridge, Free Radicals in Biology and Medicine, The Clarendon Press, Oxford University Press, New York, 1985. 


\section{Figure legends.}

Figure 1. A) Synthesis (left panel) and spectral characteristics (right panel) of FLTX1. B). FLTX1 and ER $\alpha$ colocalize in MCF7 breast cancer cells. To investigate the potential colocalization of these two molecules, we performed immunocytochemical assays using standard protocols described previously [5]. MCF7 cells were incubated with FLTX1 (green fluorescence), followed by incubation with MC20, an anti-ER $\alpha$ specific antibody (red fluorescence). The results obtained by confocal microscopy revealed that both fluorescent signals (merged images FLTX1 and ER $\alpha$ in panel 'Merge'). In the rightmost panel, transmission image overlapped with white spots corresponding to FLTX1 and MC20 colocalization.

Figure 2. Docking of A) Tamoxifen and B) FLTX1 into the binding site of ER $\alpha$ (PDB 3ERT).

Figure 3. Decay curves at different concentration of FLTX1 dissolved in acetone. IRF: Instrumental Response Function

Figure 4. Amplified Spontaneous Emission of FLTX1. A) Experimental setup; B) Narrowing of the emission spectrum as a function of the pump density; C) ASE threshold and efficiency as a function of the FLTX1 concentration.

Figure 5. Whispering Gallery Mode Laser of FLTX1. A) Laser emission spectrum of the WGMs; B) Emission intensity as a function of pump energy density.

Figure 6. Random Laser properties of FLTX1. Emission spectra as a function of pump density showing the transition from spontaneous emission to $A$ ) incoherent RL and B) coherent RL. C) Confocal microphotographs of mouse uterine wall showing the different cell layers (el: epithelial lining; st: stroma; ec: columnar epithelial cells; mg: mucous gland) labelled with FLTX1 solutions. Illustrated are, from left to right, FLTX1, propidium iodide, merged and phase contrast transmission images.

Figure 7. A) Absorbance spectra of formazan produced after irradiating a $50 \mu \mathrm{M}$ FLTX-NBT solution @ 475 $\mathrm{nm}$ at different times (Left). B) Typical absorbance curves with respect to irradiated NBT as control (Right). 